Che - . . .

Simple method for compensation of thermally-induced birefringence in high-power solid-state lasers

\author{
W. A. Clarkson, N. S. Felgate and D. C. Hanna \\ Optoelectronics Research Centre \\ University of Southampton \\ Southampton, SO17 1BJ \\ United Kingdom \\ Tel. +44 1703593776 \\ Fax. +441703 593142 \\ e-mail:wac@orc.soton.ac.uk
}

\begin{abstract}
We demonstrate that a single quarter-wave plate can be used to greatly reduce the effects of thermally-induced stress-birefringence for a diode-bar-end-pumped Nd:YAG laser operating at $946 \mathrm{~nm}, 2.9 \mathrm{~W}$ of linearly polarised output with $\mathrm{M}^{2}<1.2$ was obtained for $14.3 \mathrm{~W}$ of incident pump power.
\end{abstract}




\title{
Simple method for compensation of thermally-induced birefringence in high-power solid-state lasers
}

\author{
W. A. Clarkson, N. S. Felgate and D. C. Hanna \\ Optoelectronics Research Centre, University of Southampton, Southampton, SO17 1BJ, U.K. \\ Tel. +44 1703 593776, Fax. +44 1703 593142, e-mail: wac@orc.soton.ac.uk
}

Progress in power-scaling diode-pumped lasers has been hindered by thermal effects; notably thermal lensing and stress-birefringence. For applications requiring a linearly polarised output (e.g. nonlinear frequency conversion and Q-switching), the most serious problem is the depolarisation loss resulting from stress-induced birefringence which dramatically reduces the efficiency of low gain lasers at high pump powers. Various techniques for compensating stress-induced birefringence have been described (e.g. [1],[2]), but which are not well-suited for low gain end-pumped lasers employing a single laser rod.

In this paper we describe an alternative technique for reducing the effects of stress-induced birefringence, which has been applied to a $946 \mathrm{~nm}$ Nd:YAG laser end-pumped by a $20 \mathrm{~W}$ diode-bar. Our approach utilises only a single quarter-wave plate located between the pump incoupling mirror and the Nd:YAG rod (fig. 1). The quarter-wave plate is aligned with its fast or slow axis paraliel to the desired plane of laser polarisation, in this case defined by a Brewster plate polariser. The principle of operation can be explained by considering the transit of linearly polarised light from the polariser to the pump incoupling mirror and back to the polariser. Rays which propagate along the laser rod in the $x-z$ or $y-z$ planes will not experience any change in polarisation state, since the radial and tangential components of stress are parallel or perpendicular to the $x, y$ axes for these rays. The rays which experience the largest change in polarisation state, and hence loss at the polariser, propagate along planes inclined $45^{\circ}$ to the $\mathrm{x}-\mathrm{z}$ and $\mathrm{y}-\mathrm{z}$ planes, where the radial and tangential stress components are inclined at $45^{\circ}$ to the $x, y$ axes. With the quarter-wave plate present in the cavity, the radial and tangential polarisation components, after a single-pass of the laser rod, will subsequently be rotated by $90^{\circ}$ on double-passing the quarter-wave plate, thus emerging after a second transit of the laser rod, with no net change in the polarisation state, hence no loss at the polariser. Some degree of birefringence compensation will also occur for rays propagating along other sectors of the laser rod, with the overall result that depolarisation loss is substantially reduced.

To demonstrate the effectiveness of this technique we compared performances of our diode-barpumped $946 \mathrm{~nm} \mathrm{Nd}$ :YAG laser with and without the quarter-wave plate. Without the quarter-wave plate the maximum linearly polarised output was limited by stress-birefringence to $\sim 2 \mathrm{~W}$. In contrast, with the quarter-wave plate present the maximum output power was $\sim 2.9 \mathrm{~W}$, limited only by the available pump power of $14.3 \mathrm{~W}$ incident on the laser rod. The output beam was also nearly diffraction-limited with $M^{2}<1.2$. A measurement of the power reflected from the Brewster plate indicated that the contribution to cavity loss arising from stress-induced birefringence was $<0.001 \%$, compared with a depolarisation loss of $1.7 \%$ without the quarter-wave plate. These results clearly demonstrate the potential of this simple technique for compensation of stress-induced birefringence, which should facilitate further power-scaling of a various end-pumped lasers.

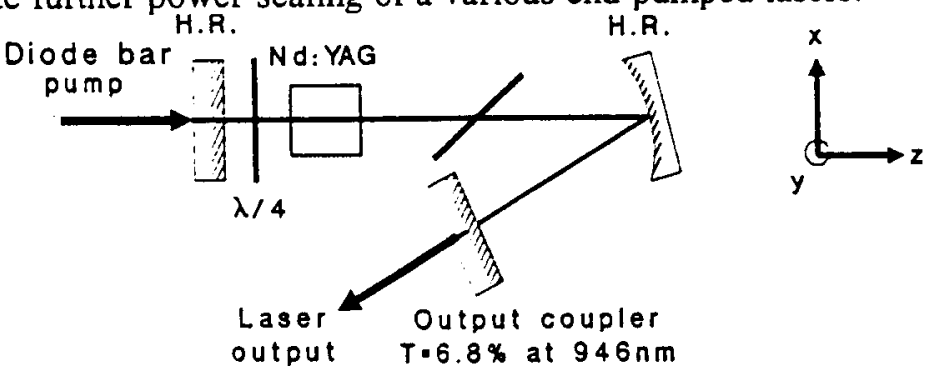

Fig. 1 Diode-pumped Nd:YAG laser with a quarter-wave plate for compensating birefringence.

\section{References}

1. W. C. Scott and M. de Wit, Appl. Phys. Lett., 18, 3, (1971).

2. W. Koechner, Solid-State Laser Engineering, Springer-Verlag, New York, p.409, (1996). 